\title{
Boundary uniqueness of harmonic functions and spectral subspaces of operator groups
}

\author{
Alexander Borichev and Yuri Tomilov
}

\begin{abstract}
We obtain new uniqueness theorems for harmonic functions defined on the unit disc or in the half plane. These results are applied to obtain new resolvent descriptions of spectral subspaces of polynomially bounded groups of operators on Banach spaces.
\end{abstract}

\section{Introduction}

It is a simple fact that a function $u$ harmonic on the unit disc and having zero limits at the boundary is equal to 0 . If however we replace (unrestricted) limits at the boundary by restricted limits (say, radial ones or non tangential ones), then the statement becomes false, see e.g. Proposition 1.4 below. One way to get the uniqueness in this case is to impose certain growth conditions on $u$.

To get an intuition on what kind of restrictions on $u$ can be imposed we recall two known uniqueness theorems for harmonic functions in the unit disc. Given a real-valued function $u$ continuous on the unit disc $\mathbb{D}$, we set

$$
M_{r}(u)=\max _{0 \leq \theta \leq 2 \pi} u\left(r e^{i \theta}\right), \quad 0<r<1 .
$$

Theorem 1.1 (V.L. Shapiro, [35]). If $u$ is harmonic in $\mathbb{D}$ and

$$
M_{r}(|u|)=o\left((1-r)^{-2}\right), \quad r \rightarrow 1-, \quad \lim _{z=r e^{i \varphi} \rightarrow e^{i \varphi}} u(z)=0, \quad \varphi \in[0,2 \pi],
$$

then $u=0$.

Theorem 1.2 (F. Wolf, Section 7 of [39]). Let $k>0$. If $u$ is harmonic in $\mathbb{D}$ and

$$
\begin{gathered}
\log ^{+} M_{r}(|u|)=o\left((1-r)^{-\pi /(2 \arctan (1 / k))}\right), \quad r \rightarrow 1-, \\
\lim _{z=r e^{i \theta} \rightarrow e^{i \varphi},|\varphi-\theta|<k(1-r)} u(z)=0, \quad \varphi \in[0,2 \pi],
\end{gathered}
$$

then $u=0$.

Mathematics Subject Classification (2010): Primary 31A20; Secondary 47D06.

Keywords: Uniqueness theorems, harmonic functions, approach regions, spectral subspaces, operator groups. 
Both results are the best possible as far as the growth assumptions on $u$ are concerned, see e.g. [8]. On the other hand, it is natural to ask for which approach domains the polynomial limit growth of $|u|$ with respect to $(1-r)^{-1}$ in the above results can be replaced by the exponential one; or more generally, what is the relation between the size of the approach domain and the limit growth of $|u|$ in boundary uniqueness theorems for harmonic $u$. In a different situation, a relation between the (Dirichlet spaces) smoothness and the boundary limits along tangential approach domains was observed in [31].

Note that as a consequence of the results in [33] and [7], in the situations we consider here, it is sufficient to impose growth restrictions just on $M_{r}(u)$. Next we define a scale of approach domains. We say that a non-decreasing continuous function $h:[0,1] \rightarrow[0,1], h(0)=0$, is an approach function. Given an approach function $h$ consider $\Delta^{h}=\{x+i y:|x| \leq h(y), 0<y<1\} \subset \mathbb{C}_{+}=\{x+i y \in \mathbb{C}: y>0\}$. Given $\varphi \in[0,2 \pi]$, the function $f_{\varphi}: z \mapsto e^{i \varphi}(i-z) /(i+z)$ maps $\mathbb{C}_{+}$onto the unit disc. Set $\Omega^{h}(\varphi)=f_{\varphi}\left(\Delta^{h}\right)$. The following two theorems give a partial answer to the above question in the case of polynomial type approach domains.

Theorem 1.3. Let $u$ be harmonic in the unit disc $\mathbb{D}$,

$$
\lim _{z \in \Omega^{h}(\varphi), z \rightarrow e^{i \varphi}} u(z)=0, \quad \varphi \in[0,2 \pi] .
$$

(a) (V.L. Shapiro) If $h(t)=0$ and

$$
M_{r}(u)=o\left((1-r)^{-2}\right), \quad r \rightarrow 1-,
$$

then $u=0$.

(b) If $h(t)=c t^{3}, c>0$, and

$$
\log ^{+} M_{r}(u)=o\left((1-r)^{-1}\right), \quad r \rightarrow 1-,
$$

then $u=0$.

(c) (F. Wolf) If $h(t)=c t, c>0$, and

$$
\log ^{+} M_{r}(u)=o\left((1-r)^{-\pi /(2 \arctan (1 / c))}\right), \quad r \rightarrow 1-,
$$

then $u=0$.

(d) If $h(t)=t^{\gamma}, 0<\gamma<1$, and

$$
\log ^{+} \log ^{+} M_{r}(u)=o\left((1-r)^{\gamma-1}\right), \quad r \rightarrow 1-,
$$

then $u=0$.

Of course, the result in (c) remains valid if $h(t)=c t+c_{1} t^{2}$ for small $t$ and for some $c_{1} \in \mathbb{R}$.

The statements of Theorem 1.3 are sharp with respect to the polynomial scale of approach domains. 
Proposition 1.4. (a) If $h(t)=o\left(t^{3}\right), t \rightarrow 0$, then there exists a function $u \neq 0$ harmonic in $\mathbb{D}$ and satisfying (1.1) such that

$$
M_{r}(|u|)=O\left((1-r)^{-2}\right), \quad r \rightarrow 1-.
$$

(b) If $1<\gamma \leq 3, h(t)=t^{\gamma}, \varepsilon>0$, then there exists a function $u \neq 0$ harmonic in $\mathbb{D}$ and satisfying (1.1) such that

$$
\log ^{+} M_{r}(|u|) \leq \varepsilon(1-r)^{-1}, \quad r \in(0,1) .
$$

(c) If $h(t)=c t, c>0, \varepsilon>0$, then there exists a function $u \neq 0$ harmonic in $\mathbb{D}$ and satisfying (1.1) such that

$$
\log ^{+} M_{r}(|u|) \leq \varepsilon(1-r)^{-\pi /(2 \arctan (1 / c))}, \quad r \in(0,1) .
$$

(d) If $0<\gamma<1, h(t)=t^{\gamma}$, then for some $k=k(\gamma)$ there exists a function $u \neq 0$ harmonic in $\mathbb{D}$ and satisfying (1.1) such that

$$
\log ^{+} \log ^{+} M_{r}(|u|) \leq k(1-r)^{\gamma-1}, \quad r \in(0,1) .
$$

Next we pass to an application of our function-theoretical results (in their halfplane version) to the study of spectral properties of operator groups on Banach spaces.

Let $X$ be a Banach space, and let $(T(t))_{t \in \mathbb{R}}$ be a $C_{0}$-group on $X$ with generator $A$ growing at most polynomially, i.e. such that $\|T(t)\| \leq M\left(1+|t|^{a}\right), a \geq 0$. There is an extensive literature on spectral properties of polynomially growing (and, even more generally, non quasianalytic) $C_{0}$-groups on Banach spaces, see e.g. $[2],[3],[5]$, [17]-[23], [27], [28]. However, the problem of description of spectral maximal subspaces of the groups (or equivalently, of the generators) in terms of nontangential boundary behavior of local resolvents of their generators has not been addressed so far (see, though, [4] and [18]). On the other hand, such characterizations look very natural being interpreted as the study of regularity of operator-valued distributions (measures) on the real line by means of their Poisson integrals.

Denote by $\sigma(x)$ the local spectrum of $x$ (i.e. the complement of the set of points $\lambda$ such that the local resolvent $R(\lambda, A) x=(\lambda-A)^{-1} x$ is analytic in a neighborhood of $\lambda$ ). For general information on the local spectra see [25], [30]. Let

$$
X(F):=\{x \in X: \sigma(x) \subset i F\}
$$

be the spectral (maximal) subspace of $A$ (equivalently of $(T(t))_{t \in \mathbb{R}}$, see, for instance, [17]) corresponding to the closed subset $F$ of $\mathbb{R}$, and let $R(\lambda, A):=(\lambda-A)^{-1}$ be the resolvent of $A$ defined at least on $\mathbb{C} \backslash i \mathbb{R}$. Recall that if $f$ belongs to the space of Schwartz test functions $\mathcal{S}(\mathbb{R})$, then

$$
\int_{-\infty}^{\infty} e^{-\alpha|t|} \hat{f}(t) T(t) d t=\int_{-\infty}^{\infty} f(\beta)(R(\alpha+i \beta, A)-R(-\alpha+i \beta, A)) d \beta,
$$

where $\hat{f}(t):=\int_{\mathbb{R}} e^{-i s t} f(s) d s$ and the integrals converge in the strong sense. 
Hence the operator-valued distribution

$$
\begin{aligned}
E & : \mathcal{S}(\mathbb{R}) \mapsto \mathcal{L}(X), \\
\langle E, f\rangle & :=\lim _{\alpha \rightarrow 0+} \int_{\mathbb{R}} f(\beta)(R(\alpha+i \beta, A)-R(-\alpha+i \beta, A)) d \beta=\int_{\mathbb{R}} \hat{f}(t) T(t) d t
\end{aligned}
$$

is well-defined.

Let $x$ be such that $\sigma(x)$ is compact, and $E_{x}:=E(\cdot) x$. Then, denoting

$$
D(\alpha+i \beta)=: R(\alpha+i \beta, A)-R(-\alpha+i \beta, A), \quad \alpha \neq 0,
$$

and following the observation in page 139 of [18], we obtain that

$$
D(\alpha+i \beta) x=2 \pi\left\langle E_{x}, \frac{1}{\pi} \frac{\alpha}{\alpha^{2}+(\beta-\cdot)^{2}}\right\rangle=2 \pi\left(E_{x} * P_{\alpha}\right)(\beta),
$$

where (1.2) is understood as the distributional convolution of a distribution having compact support with a $C^{\infty}(\mathbb{R})$-function. (Remark also that

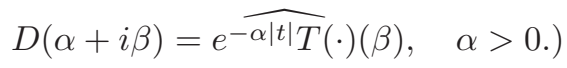

Thus $D(\cdot) x$ can be treated as the Poisson integral of $E_{x}$. (Using distributions in $\mathcal{D}_{L_{p}}^{\prime}$ one can write down the Poisson integral of $E_{x}$ without any restrictions on $\sigma(x)$, see e.g. [1]. This however would unnecessarily complicate the presentation.)

If $X$ is a Hilbert space with inner product $(\cdot, \cdot)$ and $(T(t))_{t \geq 0}$ is a unitary $C_{0}$-group on $X$ so that $-i A$ is self-adjoint, then the distribution $E$ can be identified with a multiple of the spectral measure $E(\cdot)$ of $-i A$, and the relation $(1.2)$ can be interpreted using the usual convolution. In particular,

$$
(D(\alpha+i \beta) x, x)=2 \int_{-\infty}^{\infty} \frac{\alpha d(E(t) x, x)}{\alpha^{2}+(\beta-t)^{2}}, \quad x \in X .
$$

It is well-known that the smoothness of a (finite) measure $\mu$ determines the boundary behavior of its Poisson integral $\mu * P_{\alpha}$. On the other hand, in many cases the radial convergence of $\mu * P_{\alpha}$ may imply certain regularity of $\mu$, see [26], [16], [12], and [10] for a general account of Tauberian results of this type.

For instance, if one has in (1.3)

$$
\lim _{\alpha \rightarrow 0+}(D(\alpha+i \beta) x, x)=0, \quad \beta \in(a, b),
$$

then $E((a, b))=0$, see for example [26].

Thus, in the case of unitary $(T(t))_{t \in \mathbb{R}}$, one easily obtains the description of spectral subspaces of $A$ (or equivalently of $(T(t))_{t \in \mathbb{R}}$ ) in terms of the Poisson integrals $E * P_{\alpha}$ :

$$
X(F)=\left\{x \in X: \lim _{\alpha \rightarrow 0+} D(\alpha+i \beta) x=0, \beta \in \mathbb{R} \backslash F\right\} .
$$


Furthermore, if the point spectrum $\sigma_{p}(A)$ of $A$ is empty, then

$$
X(F)=\left\{x \in X: \sup _{\alpha>0}\|D(\alpha+i \beta) x\|<\infty, \beta \in \mathbb{R} \backslash F\right\},
$$

cf. Theorem 1.5 below. Such descriptions are of value, for instance, in scattering theory, see e.g. Chapter 3 of [5].

If $X$ is a Banach space and/or $C_{0}$-group $(T(t))_{t \in \mathbb{R}}$ on $X$ is polynomially bounded, then $E$ is merely a distribution although one still has the formula (1.2). In this setting one could not expect in general direct relations between the boundary behavior of $D$ and the regularity of $E$. Nevertheless, it appears that if $(T(t))_{t \in \mathbb{R}}$ does not grow too fast and the Poisson integral of $E$ has zero radial boundary values, then $E$ is forced to be zero, thus a distributional counterpart of tauberian theorems in [26], [16] and [34] holds. More precisely, the following statement is true.

Theorem 1.5. Let $X$ be a Banach space, and let $(T(t))_{t \in \mathbb{R}}$ be a $C_{0}$-group on $X$ with generator $A$, such that $\|T(t)\| \leq M\left(1+|t|^{a}\right), a \in[0,2]$. Then, for any closed $F \subset \mathbb{R}$,

$$
X(F)=\left\{x \in X: \lim _{\alpha \rightarrow 0+} D(\alpha+i \beta) x=0, \beta \in \mathbb{R} \backslash F\right\} .
$$

If $X$ is reflexive, $\sigma_{p}(A)=\emptyset$ and $a \in[0,2)$, then moreover

$$
X(F)=\left\{x \in X: \sup _{\alpha>0}\|D(\alpha+i \beta) x\|<\infty, \beta \in \mathbb{R} \backslash F\right\} .
$$

Note that we require neither UMD property of the underlying Banach space, nor the boundedness of the group. Thus a well-developed spectral theory of (mostly) bounded groups on UMD spaces is by no means available in our situation.

Theorem 1.5 can be used to give an algebraic description of spectral subspaces of $(T(t))_{t \in \mathbb{R}}$, thus partially improving and extending Theorem 3.1 of [13] (see also Theorem 3.2 and Remark 3.5 in [14], Theorem 1.2 in [37] and Theorem 4 in [29]) to linear (in general, unbounded) operators generating $C_{0}$-groups of subquadratic growth. For a related result on the generators of bounded groups, see Corollary 6.7 of [4]. Let $\operatorname{ran}(A)$ stand for the range of an operator $A$.

Theorem 1.6. Let $X$ be a Banach space, and let $(T(t))_{t \in \mathbb{R}}$ be a $C_{0}$-group on $X$ with generator $A$, such that $\|T(t)\| \leq C\left(1+|t|^{a}\right)$.

If $a \in[0,1)$, then for any closed $F \subset \mathbb{R}$,

$$
X(F)=\bigcap_{\beta \in \mathbb{R} \backslash F} \operatorname{ran}(i \beta-A)^{2} .
$$

If $a \in[1,2)$, then for any closed $F \subset \mathbb{R}$,

$$
X(F)=\bigcap_{\beta \in \mathbb{R} \backslash F} \operatorname{ran}(i \beta-A)^{3} .
$$


Remark 1.7. Note that the right hand side of (1.6) does not depend on the exponent $a$ while the results in [14], [37], [13], [29] formulated (for in general unbounded operators) as in Theorem 1.6 would only yield

$$
X(F)=\bigcap_{\beta \in \mathbb{R} \backslash F} \operatorname{ran}(i \beta-A)^{a+2} .
$$

We prove Theorem 1.3 in Section 2. The (counter)-examples (Proposition 1.4) are discussed in Section 3. In Section 4 we formulate a maximum principle and a local half plane versions of Theorem 1.3. Finally, in Section 5 we prove Theorems 1.5 and 1.6.

\section{The proof of Theorem 1.3}

First of all, using Section 1.3 of [33] or [7], we replace conditions on $M_{r}(u)$ by the same conditions on $M_{r}(|u|)$.

The scheme of our proof goes back at least to F. Wolf (1941). Let us denote by $R$ the set of $\theta \in[0,2 \pi]$ such that $u$ is continuous in a neighborhood of $e^{i \theta}$ (in the unit disc). Then $S=[0,2 \pi] \backslash R$ is a closed subset of $[0,2 \pi]$.

Let us suppose that $S$ is not empty (without loss of generality, $S \neq\{0,2 \pi\}$ ), and denote

$$
S_{N}=\left\{\varphi: \sup _{z \in \Omega^{h}(\varphi)}|u(z)| \leq N\right\} .
$$

Then the sets $S_{N}$ are closed, and by a Baire category type argument, we can find an open interval $I \subset[0,2 \pi]$ and $N<\infty$ such that

$$
S \cap I=S_{N} \cap I \neq \emptyset,
$$

and, hence,

$$
\sup _{z \in \Omega^{h}(\varphi), \varphi \in S \cap I}|u(z)| \leq N .
$$

It remains to prove that for any closed interval $J \subset I, u$ is bounded in the sector $W_{J}=\left\{r e^{i \theta}: 0 \leq r<1, \theta \in J\right\}$. After that, standard uniqueness results give us that $u$ is continuous up to $\left\{e^{i \theta}: \theta \in J\right\}$ and vanishes there. This contradicts to (2.1), and, hence, to the assumption that $S$ is not empty. Thus, our proof will be completed.

Note that $|u|$ is bounded by $N$ on $\cup_{\varphi \in S \cap J} \Omega^{h}(\varphi)$ and is bounded near any closed subinterval of $R \cap J$. Let $J_{1} \subset R \cap J$ be an open interval such that its endpoints $\varphi_{1}, \varphi_{2}$ are in $S$, and let $T$ be the connected component of $\left\{r e^{i \theta}: 1 / 2<r<1\right.$, $\left.\theta \in J_{1}\right\} \backslash\left(\Omega^{h}\left(\varphi_{1}\right) \cup \Omega^{h}\left(\varphi_{2}\right)\right)$ such that $\partial T \cap \partial \mathbb{D} \neq \emptyset$.

It remains to verify that $u$ is bounded in every such $T$. After that, applying the maximum principle,

$$
\sup _{T}|u| \leq \sup _{\Omega^{h}\left(\varphi_{1}\right) \cup \Omega^{h}\left(\varphi_{2}\right) \cup D(0,1 / 2)}|u|
$$

(where $D(z, R)=\{w:|w-z| \leq R\}$ ), we obtain that $|u|$ is bounded in every $T$ by $\max \left[N, \sup _{D(0,1 / 2)}|u|\right]$, and, hence, bounded in $W_{J}$. 
Thus, after applying a conformal map onto the upper half-plane $\mathbb{C}_{+}$, our problem is reduced to the following one.

Given a function $u$ harmonic in $Q=\{x+i y:|x|<1,0<y<1\}$, denote

$$
M_{y}=\max _{0<x<1}|u(x+i y)| .
$$

Reduced problem. Suppose that $u$ is continuous up to $(0, \delta) \subset \partial Q, u=0$ on $(0, \delta)$ for some $\delta>0$ and $u$ is bounded on $\Delta^{h} \cap Q$.

If (a) $h(t)=0$ and

$$
M_{y}=o\left(y^{-2}\right), \quad y \rightarrow 0+,
$$

or (b) $h(t)=c t^{3}, c>0$, and

$$
\log ^{+} M_{y}=o\left(y^{-1}\right), \quad y \rightarrow 0+,
$$

or $(\mathrm{c}) h(t)=c t, c>0$, and

$$
\log ^{+} M_{y}=o\left(y^{-\pi /(2 \arctan (1 / c))}\right), \quad y \rightarrow 0+,
$$

or $(\mathrm{d}) h(t)=t^{\gamma}, 0<\gamma<1$, and

$$
\log ^{+} \log ^{+} M_{y}=o\left(y^{\gamma-1}\right), \quad y \rightarrow 0+,
$$

then $u$ is bounded in a neighborhood of the point 0 in $\{x+i y \in Q: x>0\}$.

Here $\varphi_{1}$ corresponds to $0, \Omega^{h}\left(\varphi_{1}\right)$ corresponds to $\Delta^{h}$, and a subinterval of $\left(\varphi_{1}, \varphi_{2}\right)$ with endpoint at $\varphi_{1}$ corresponds to $(0, \delta)$. The function $u$ is bounded in every domain $\{z \in Q: 0<\varepsilon<|z|<\delta\}$, and we need to verify that $u$ is bounded near the point 0 .

Next we apply the Schwartz reflection principle and then consider the function $v(z)$ equal to $u(1 / z)$ in the cases (a) and (b), equal to

$$
u\left(z^{-(2 / \pi) \arctan (1 / c)}\right)
$$

in the case (c) and equal to

$$
u\left(\left(\frac{2(1-\gamma)}{\pi \gamma} \log z\right)^{\gamma /(\gamma-1)}\right)
$$

in the case (d) (where we choose the principal branch of $\log z$ ).

Then the interval $(0, \delta)$ in the reduced problem becomes $\left(c_{\delta},+\infty\right)$, the function $v$ is harmonic in $O=\mathbb{C} \backslash((-\infty, 0] \cup K)$ for some compact $K, v=0$ on $O \cap(0, \infty)$,

$$
\begin{gathered}
\lim _{|y| \rightarrow \infty,-\varphi(y) \leq x \leq 0} v(x+i y)=0, \\
\left|v\left(r e^{i \theta}\right)\right| \leq \psi(r, \theta), \quad-\pi / 2 \leq \theta \leq \pi / 2, r>0,
\end{gathered}
$$

for certain functions $\varphi, \psi$, and we need only to verify that $v$ is bounded at infinity in the right half-plane $\Pi$. 
Here in the case (a) $\varphi=0, \psi(r, \theta)=o\left(r^{2} /|\theta|^{2}\right), r \rightarrow \infty$, in the case (b) $\varphi(y)=k /|y|, \log ^{+} \psi(r, \theta)=o(r /|\theta|), r \rightarrow \infty$, in the case (c) $\varphi(y)=k|y|$, $\log ^{+} \psi(r, \theta)=o\left(r /|\theta|^{k_{1}}\right), r \rightarrow \infty$, and in the case $(\mathrm{d}) \varphi(y)=|y|, \log ^{+} \log ^{+} \psi(r, \theta)=$ $o\left(\log r /|\theta|^{1-\gamma}\right), r \rightarrow \infty$, for some positive numbers $k, k_{1}$ depending on $c$. (In fact, in the case $(\mathrm{d})$, the property $(2.2)$ holds for any positive $\varphi$.)

To get rid of the singularities in the estimates (2.3) we use the log-log theorem of Levinson-Sjöberg. Namely, we consider the functions $v_{n}, v_{n}(z)=v\left(2^{n} z\right)$. The functions $f_{n}$ analytic on $H=\{x+i y: 1 / 2 \leq x \leq 4,|y| \leq 2\}$ are determined by the relations $\operatorname{Re} f_{n}=v_{n}, f_{n}(1+i)=v_{n}(1+i)$.

We use the fact that given a function $v$ harmonic in the disc $D(z, R)$ and its conjugate function $\tilde{v}$, for an absolute positive constant $c$ we have

$$
\|\operatorname{grad} \tilde{v}(z)\| \leq \frac{c}{R} \sup _{w_{1}, w_{2} \in D(z, R)}\left|u\left(w_{1}\right)-u\left(w_{2}\right)\right| .
$$

Then the functions $f_{n}$ satisfy the following estimates on $H$ :

$$
\begin{aligned}
& \text { (a) }\left|f_{n}(x+i y)\right|=\frac{o\left(2^{2 n}\right)}{|y|^{2}}, \quad n \rightarrow \infty, \\
& \text { (b) } \log ^{+}\left|f_{n}(x+i y)\right|=\frac{o\left(2^{n}\right)}{|y|}, \quad n \rightarrow \infty, \\
& \text { (c) } \log ^{+}\left|f_{n}(x+i y)\right|=\frac{o\left(2^{n}\right)}{|y|^{k_{1}}}, \quad n \rightarrow \infty, \\
& \text { (d) } \log ^{+} \log ^{+}\left|f_{n}(x+i y)\right|=\frac{o(n)}{|y|^{1-\gamma}}, \quad n \rightarrow \infty .
\end{aligned}
$$

Next, we apply the following quantitative version of the log-log theorem (see Section 3 of [11]).

Theorem 2.1 (Y. Domar, see the argument on pages 376-379 of [22]). Let $w$ : $(0,2) \rightarrow[1, \infty)$ be a decreasing function. If $h$ is subharmonic on $H, h(x+i y) \leq$ $w(|y|), x+i y \in H$, and

$$
\sum_{k \geq 0} w^{-1}\left(2^{k} T\right) \leq \frac{1}{10}
$$

for some $T>0$, then

$$
h(z) \leq 2 T, \quad z \in H_{0}=\{x+i y: 1 \leq x \leq 2,|y| \leq 1\} .
$$

Applying this theorem to $\log ^{+}\left|f_{n}\right|$ we obtain

$$
\begin{aligned}
& \text { (a) } \sup _{H_{0}}\left|v_{n}\right|=o\left(2^{2 n}\right), \quad n \rightarrow \infty, \\
& \text { (b)-(c) } \sup _{H_{0}} \log ^{+}\left|v_{n}\right|=o\left(2^{n}\right), \quad n \rightarrow \infty, \\
& \text { (d) } \sup _{H_{0}} \log ^{+} \log ^{+}\left|v_{n}\right|=o\left(n^{1 / \gamma}\right), \quad n \rightarrow \infty .
\end{aligned}
$$


Thus, the function $v$ satisfies the following estimates in $\Pi$ :

$$
\begin{aligned}
& \text { (a) }|v(z)|=o\left(z^{2}\right), \quad|z| \rightarrow \infty, \\
& \text { (b)-(c) } \log ^{+}|v(z)|=o(z), \quad|z| \rightarrow \infty, \\
& \text { (d) } \quad \log ^{+} \log ^{+}\left|v\left(r e^{i \theta}\right)\right|= \begin{cases}o\left(\frac{\log r}{|\theta|^{1-\gamma}}\right), & |\theta| \leq \frac{\pi}{2}, \\
o\left((\log r)^{1 / \gamma}\right), & |\theta| \leq \frac{\pi}{4},\end{cases}
\end{aligned}
$$

In the cases (b)-(d) we fix $c \in O$ and define an analytic function $f$ by the relations $\operatorname{Re} f=v, f(c)=v(c)$. Again by (2.4) we obtain that

$$
\begin{aligned}
& \text { (b)-(c) }|f(i y-a /(2|y|))|=o\left(y^{2}\right), \quad|y| \rightarrow \infty, \\
& \text { (b)-(c) } \log ^{+}|f(i y-s)|=o(y), \quad|y| \rightarrow \infty, 0 \leq s \leq a /(2|y|), \\
& \text { (b)-(c) } \log ^{+}|f(z)|=o(z), \quad z \in \Pi,|z| \rightarrow \infty, \\
& \text { (d) }|f(i y-1)|=o(y), \quad|y| \rightarrow \infty, \\
& \text { (d) } \log ^{+}|f(i y-s)|=o(y), \quad|y| \rightarrow \infty, 0 \leq s \leq 1, \\
& \text { (d) } \log ^{+} \log ^{+}\left|f\left(r e^{i \theta}\right)\right|=\left\{\begin{array}{l}
o\left(\frac{\log r}{|\theta|^{1-\gamma}}\right), \quad|\theta| \leq \frac{\pi}{2}, \quad r \rightarrow \infty, \\
o\left((\log r)^{1 / \gamma}\right), \quad|\theta| \leq \frac{\pi}{4},
\end{array}\right.
\end{aligned}
$$

where $a=k$ in the case (b) and $a=1$ in the case (c).

By a Phragmén-Lindelöf type theorem (using the argument of [22], III C), in the case (b)-(c) we obtain that

$$
|f(z)|=o\left(z^{2}\right), \quad z \in \Pi,|z| \rightarrow \infty .
$$

In the case (d), for a sufficiently large closed disc $U=\overline{D(0, R)}$, we apply the following lemma to the function $w(z)=\log ^{+}\left|f(z-1) /(z+1)^{2}\right|$, subharmonic in $\Omega=\Pi \backslash U$, where $\Pi$ is the right half plane.

Lemma 2.2. Let $w$ be subharmonic in $\Omega$ and upper semicontinuous in $\bar{\Omega}, w \leq 0$ on $\partial \Omega, 0<\gamma<1$, and let

$$
\log ^{+}\left|w\left(r e^{i \theta}\right)\right|=\left\{\begin{array}{cl}
o\left(\frac{\log r}{|\theta|^{1-\gamma}}\right), & |\theta| \leq \frac{\pi}{2}, \\
o\left((\log r)^{1 / \gamma}\right), & |\theta| \leq \frac{\pi}{4},
\end{array}\right.
$$

Then $w \leq 0$ in $\Omega$.

Proof. First of all we choose $\delta>0$ such that

$$
\exp \left[(n-1)^{2}\right] \geq 2 \delta n \exp \left[(1-\gamma)(n+1)^{2}\right], \quad n \geq 1
$$


We fix $z \in \Omega$. For large $N, M$ we consider the function

$$
\beta(x)=\left\{\begin{array}{c}
e^{N-1}, \quad 0 \leq x \leq e^{N}, \\
x \exp \left[-\left(\frac{\log x}{N}\right)^{2}\right], \quad x>e^{N},
\end{array}\right.
$$

the domain

$$
\Sigma=\left\{x+i y \in \Omega: 0<x<e^{N M},|y|<\beta(x)\right\}
$$

and the sets

$$
\begin{aligned}
S_{0} & =\left\{x+i y \in \partial \Sigma: 0<x<e^{N}\right\}, \\
S_{n} & =\left\{x+i y \in \partial \Sigma: e^{n N}<x<e^{(n+1) N}\right\}, \quad 1 \leq n<M, \\
S_{M} & =\left\{x+i y \in \partial \Sigma: x=e^{N M}\right\} .
\end{aligned}
$$

Then by the theorem on harmonic estimation (see page 256 in [22]) we have

$$
u(z) \leq \sum_{0 \leq n \leq M} \omega\left(z, S_{n}, \Sigma\right) \cdot \sup _{S_{n}} w
$$

where $\omega(z, S, \Sigma)$ is harmonic measure of $S \subset \partial \Sigma$ with respect to $z$ in $\Sigma$.

An easy geometric argument shows that for $N \geq N(z)$ we have

$$
\omega\left(z, S_{0}, \Sigma\right)+\omega\left(z, S_{1}, \Sigma\right) \leq e^{-N / 2} .
$$

Indeed, it suffices to estimate the left hand side expression from above by

$$
\begin{aligned}
& \omega\left(z, \partial D\left(0, e^{N-1}\right) \cap \Pi, D\left(0, e^{N-1}\right) \cap \Pi\right)= \\
& \quad=\omega\left(z e^{1-N}, \partial D(0,1) \cap \Pi, D(0,1)\right)-\omega\left(z e^{1-N}, \partial D(0,1) \backslash \Pi, D(0,1)\right) \\
& \quad \asymp c|z| e^{-N}, \quad N \rightarrow \infty .
\end{aligned}
$$

Furthermore, the Ahlfors-Carleman theorem (see, for instance, Theorem 6.1 in page 148 of [15]) shows that for large $N$ we have

$$
\begin{aligned}
\omega\left(z, S_{n}, \Sigma\right) & \leq \frac{8}{\pi} \exp \left(-\pi \int_{\exp ((n-1) N)}^{\exp (n N)} \frac{d r}{r \exp \left[-\left(\frac{\log r}{N}\right)^{2}\right]}\right) \\
& \leq \exp \left[-N e^{(n-1)^{2}}\right], \quad 2 \leq n \leq M
\end{aligned}
$$

Next we use that by (2.7), for large $N \geq N(\delta)$, the following estimates are fulfilled:

$$
\begin{aligned}
\sup _{S_{0} \cup S_{1}} w & \leq e^{N / 2} \\
\sup _{S_{n}} w & \leq \exp \left[\delta n N \exp \left[(1-\gamma)(n+1)^{2}\right]\right], \quad 1<n<M, \\
\sup _{S_{M}} w & \leq \exp \left[(N M)^{1 / \gamma}\right] .
\end{aligned}
$$


By (2.9), we obtain that

$$
\begin{aligned}
w(z) \leq 1 & +\exp \left[(N M)^{1 / \gamma}-N e^{(M-1)^{2}}\right] \\
& +\sum_{2 \leq n<M} \exp \left[\delta n N \exp \left[(1-\gamma)(n+1)^{2}\right]-N e^{(n-1)^{2}}\right] .
\end{aligned}
$$

For large $N \geq N(\delta), M \geq M(N, \gamma)$, using (2.8) we conclude that

$$
w(z) \leq 2+\sum_{2 \leq n<M} \exp \left[-\delta n N \exp \left[(1-\gamma)(n+1)^{2}\right]\right] \leq 3 .
$$

Thus, $w$ is bounded on $\Omega$, and hence, $w \leq 0$ on $\Omega$.

As a result, in the case (d) we obtain

$$
|f(z)|=O\left(z^{2}\right), \quad z \in \Pi,|z| \rightarrow \infty,
$$

and the Phragmén-Lindelöf theorem together with (2.5), (2.6) gives us that

$$
|f(z)|=o(z), \quad z \in \Pi,|z| \rightarrow \infty .
$$

Finally, in the cases (a)-(d) the harmonic function $v$ is bounded at infinity on $i \mathbb{R} \cup \mathbb{R}_{+}$and

$$
|v(z)|=o\left(z^{2}\right), \quad z \in \Pi,|z| \rightarrow \infty .
$$

Therefore, $v$ is bounded in $\Pi$ and our proof is completed.

\section{Examples}

Proof of Proposition 1.4. (a) If $h(t)=o\left(t^{3}\right), t \rightarrow 0$, then the function $u\left(r e^{i \theta}\right)=$ $\sum_{n>0} n r^{n} \sin (n \theta)$ is harmonic in $\mathbb{D}$,

$$
M_{r}(u)=O\left((1-r)^{-2}\right), \quad r \rightarrow 1-,
$$

and

$$
\lim _{z \in \Omega^{h}(\varphi), z \rightarrow e^{i \varphi}} u(z)=0, \quad \varphi \in[0,2 \pi],
$$

Next, we use the construction from the Appendix of [8]. It is valid in the case (c) and works with small modifications in the cases (b), (d). For the sake of simplicity, we give here just a local half plane example in the case (b). Namely, if $1<\gamma \leq 3, h(t)=t^{\gamma}, \varepsilon>0$, then there exists a function $u \neq 0$ harmonic in $Q=\{x+i y:|x|<1,0<y<1\}$ and such that

$$
\begin{aligned}
\log ^{+}|u(x+i y)| & \leq \frac{\varepsilon}{y}, \\
\lim _{z \in Q, z \rightarrow x} u(z) & =0, \quad x \in[-1,1] \backslash\{0\}, \\
\lim _{z \in \Delta^{h}, z \rightarrow 0} u(z) & =0 .
\end{aligned}
$$


Construction. Let $\max (0,2-\gamma)<\delta<1$, and let

$$
f_{0}(z)=\exp \left(\frac{\varepsilon}{z}-\frac{1}{z^{\delta}}\right), \quad z \in \bar{Q} \backslash \Delta^{h}, \operatorname{Re} z>0,
$$

where the branch of $z^{\delta}$ is chosen to be positive on the positive half axis. Then

$$
\begin{aligned}
\operatorname{Im} f_{0}(x) & =0, \quad x \in(0,1), \\
\left|f_{0}(x+i y)\right| & \leq \exp \frac{\varepsilon}{y}, \quad x+i y \in Q, \\
\left|f_{0}(z)\right| & \leq \exp \left(-c|z|^{-\delta}\right), \quad z=x+i y, y^{\gamma}<x<2 y^{\gamma}, 0<y<1 .
\end{aligned}
$$

Next, fix $f_{1} \in C^{2}([1,2])$ with $0 \leq f_{1} \leq 1$, such that $f_{1}$ vanishes in a neighborhood of the point 1 , and equals 1 in a neighborhood of the point 2. Define $f_{2}(z)$, $z=x+i y \in Q$, by $f_{2}(z)=f_{0}(z), x>2 y^{\gamma}, f_{2}(z)=f_{0}(z) f_{1}(t), x=t y^{\gamma}, 1 \leq t \leq 2$, $f_{2}(z)=0, x<y^{\gamma}$. We have

$$
\begin{aligned}
& f_{2} \in C^{2}(\bar{Q} \backslash\{0\}), \\
& \operatorname{Im} f_{2} \notin L^{\infty}(Q), \\
& \bar{\partial} f_{2} \in L^{\infty}(Q), \\
& \lim _{z \rightarrow 0} \bar{\partial} f_{2}(z)=0, \\
& \operatorname{Im} f_{2}(z)=0, \quad z \in \Delta^{h} \cup[-1,1] \backslash\{0\}, \\
& \left|f_{2}(x+i y)\right| \leq C \exp \frac{\varepsilon}{y} .
\end{aligned}
$$

Now we define

$$
f_{3}(z)=\frac{1}{\pi} \int_{Q} \frac{\bar{\partial} f_{2}(\zeta)}{z-\zeta} d m_{2}(\zeta),
$$

where $m_{2}$ is planar Lebesgue measure. Then $f_{3} \in C(\bar{Q}) \cap C^{1}(Q)$, and $\bar{\partial} f_{3}=\bar{\partial} f_{2}$ on $Q$. Let $f_{4}$ be a function harmonic in $Q$ and continuous in $\bar{Q}$ such that $f_{4}=\operatorname{Im} f_{3}$ on $[-1,1]$. Denote

$$
u=\operatorname{Im}\left(f_{2}-f_{3}\right)+f_{4} .
$$

Then $u$ is harmonic in $Q, u \neq 0, u \in C(\bar{Q} \backslash\{0\}),\left.u\right|_{[-1,1] \backslash\{0\}}=0,|u(x+i y)| \leq$ $C+C \exp (\varepsilon / y)$, and

$$
\lim _{z \in \Delta^{h}, z \rightarrow 0} u(z)=\lim _{z \in \Delta^{h}, z \rightarrow 0}\left(f_{4}(z)-\operatorname{Im} f_{3}(z)\right)=0 .
$$

If $h$ decreases at zero slower than a power of $t$, then the critical growth rate is bigger than in Theorem $1.3(\mathrm{~d})$. However, the growth

$$
\int^{1} \log ^{+} \log ^{+} M_{r}(u) d r=\infty,
$$

does not correspond to any approach domain. One encounters the same critical growth threshold for the existence of both MacLane asymptotical and Beurling generalized distributional boundary values for analytic functions (see [9] for a discussion). 
Here we have the following result.

Remark 3.1. If $f$ is a sufficiently regular positive function on $(0, \infty)$ such that

$$
\left\{\begin{array}{c}
\lim _{x \rightarrow \infty} f(x)=\infty \\
\int^{\infty} \frac{f(x)}{x^{2}} d x=\infty
\end{array}\right.
$$

and $h$ is an approach function, then there exists a function $u \neq 0$ harmonic in $\mathbb{D}$ such that

$$
\log ^{+} \log ^{+} M_{r}(u)=O\left(f\left((1-r)^{-1}\right)\right), \quad r \rightarrow 1-,
$$

and

$$
\lim _{z \in \Omega^{h}(\varphi), z \rightarrow e^{i \varphi}} u(z)=0, \quad \varphi \in[0,2 \pi],
$$

Let us sketch a construction of such a function (cf. Example 3.3 of [6]). It suffices to find a sufficiently regular increasing function $\beta:[0,1] \rightarrow[0,1], \beta(0)=0$, such that

$$
\left\{\begin{array}{c}
\beta(h(t))=o(t), \quad t \rightarrow 0, \\
\int_{t} \frac{d s}{\beta(s)}=o(f(1 / \beta(t))), \quad t \rightarrow 0 .
\end{array}\right.
$$

If $\Omega=\{x+i y: 0<x<1,|y|<\beta(x)\}$, and $\Phi$ is a conformal map of $\Omega$ onto $\mathbb{C}_{+}$such that $\Phi(0)=\infty, \Phi((0,1))=i \mathbb{R}_{+}$, then we consider the analytic function $\exp (-i \Phi)$. The estimates by Warschawski on the asymptotics of $\Phi$ together with properties (3.2) give us a possibility to use the scheme of the Appendix of [8]. Finally, relations (3.1) guarantee the existence of $\beta$ satisfying (3.2).

\section{Maximal and local half plane versions of Theorem 1.3}

An easy modification of the argument in Section 2 gives us the following maximum principle, cf. [8], [39]. For a different set of problems in this direction see [36].

Theorem 4.1. Let $u$ be harmonic in the unit disc $\mathbb{D}$ and let

$$
\limsup _{z \in \Omega^{h}(\varphi), z \rightarrow e^{i \varphi}}|u(z)| \leq 1, \quad \varphi \in[0,2 \pi] .
$$

(a) If $h(t)=0$ and

$$
M_{r}(u)=o\left((1-r)^{-2}\right), \quad r \rightarrow 1-,
$$

then $|u| \leq 1$ in $\mathbb{D}$.

(b) If $\lim _{t \rightarrow 0} h(t) t^{-3}=\infty$ and

$$
\log ^{+} M_{r}(u)=o\left((1-r)^{-1}\right), \quad r \rightarrow 1-,
$$

then $|u| \leq 1$ in $\mathbb{D}$. 
(c) If $h(t)=c t, c>0$, and

$$
\log ^{+} M_{r}(u)=o\left((1-r)^{-\pi /(2 \arctan (1 / c))}\right), \quad r \rightarrow 1-,
$$

then $|u| \leq 1$ in $\mathbb{D}$.

(d) If $h(t)=t^{\gamma}, 0<\gamma<1$, and

$$
\log ^{+} \log ^{+} M_{r}(u)=o\left((1-r)^{\gamma-1}\right), \quad r \rightarrow 1-,
$$

then $|u| \leq 1$ in $\mathbb{D}$.

Next we formulate a local half plane version of Theorem 1.3 (a), (b) which will be needed in the next section.

Define the rectangle

$$
Q:=\{z=x+i y:-1<x<1,0<y<1\},
$$

and for every $x \in(-1,1)$ and the approach function $h$ define $\Delta^{h}(x):=\Delta^{h}+x$.

Let $M_{y}(u):=\sup _{x \in(-1,1)}|u(x+i y)|$.

Theorem 4.2. Let $u$ be a harmonic function on $Q$. Assume that

$$
\lim _{z \in \Delta^{h}(x), z \rightarrow x} u(z)=0, \quad x \in[-1,1] .
$$

If either

(a) $h(t)=0$ and

$$
M_{y}(u)=o\left(y^{-2}\right), \quad y \rightarrow 0+
$$

or

(b) $h(t)=c t^{3}, c>0$, and

$$
\log ^{+} M_{y}(u)=o\left(y^{-1}\right), \quad y \rightarrow 0+,
$$

then $u$ extends continuously to the interval $(-1,1)$, and $u=0$ on $(-1,1)$.

The proof is similar to that of Theorem 1.3.

\section{An operator theoretical application}

In this section we derive Theorems 1.5 and 1.6 stated in Introduction as corollaries of our local uniqueness result, Theorem 4.2 .

If $X$ is a Banach space, and $(T(t))_{t \in \mathbb{R}}$ is a $C_{0}$-group on $X$ with the generator $A$ such that $\|T(t)\| \leq M\left(1+|t|^{a}\right), a \geq 0$, then the resolvent $R(\lambda, A), \lambda \in \mathbb{C} \backslash i \mathbb{R}$, is the Carleman transform of $(T(t))_{t \in \mathbb{R}}$,

$$
R(\lambda, A):= \begin{cases}\int_{0}^{\infty} e^{-\lambda t} T(t) d t, & \operatorname{Re} \lambda>0, \\ -\int_{-\infty}^{0} e^{-\lambda t} T(t) d t, & \operatorname{Re} \lambda<0\end{cases}
$$

thus

$$
\|R(\lambda, A)\| \leq \frac{M}{|\operatorname{Re} \lambda|^{a+1}}, \quad \operatorname{Re} \lambda \neq 0
$$


The estimate (5.2) and the resolvent identity allow us to extend the property of horizontal convergence of $D(\alpha+i \beta)=R(\alpha+i \beta, A)-R(-\alpha+i \beta, A)$ to the regions

$$
\Omega^{h}(\beta):=\left\{\alpha+i \beta^{\prime}: \alpha \in(0,1),\left|\beta-\beta^{\prime}\right| \leq h(\alpha)\right\},
$$

corresponding to the approach function $h(t)=t^{a+1}$. Let $\operatorname{dom}(A)$ denote the domain of an operator $A$.

Lemma 5.1. Let $X$ be a Banach space, and let $x \in X$ be fixed.

(i) $\lim _{\alpha \rightarrow 0+}\|D(\alpha+i \beta) x\|=0$ if and only if $\lim _{z \rightarrow i \beta, z \in \Omega^{h}(\beta)}\|D(\alpha+i \beta) x\|=0$.

(ii) If $\sigma_{p}(A)=\emptyset$, then the limit $\lim _{\alpha \rightarrow 0+} D(\alpha+i \beta) x$ exists if and only if $\lim _{\alpha \rightarrow 0+}\|D(\alpha+i \beta) x\|=0$.

(iii) If $X$ is reflexive and $\sigma_{p}(A)=\emptyset$, then $\sup _{\alpha>0}\|D(\alpha+i \beta) x\|<\infty$ if and only if $D(\alpha+i \beta) x$ tends to 0 weakly as $\alpha \rightarrow 0+$.

Proof. (i) Fix $x$ and $\beta$ such that $\lim _{\alpha \rightarrow 0+}\|D(\alpha+i \beta) x\|=0$. We use that

$$
\begin{gathered}
D(\alpha+i \beta)=2 \alpha\left[\alpha^{2}-(A-i \beta)^{2}\right]^{-1} \\
(A-i \beta) D(\alpha+i \beta)=\alpha[R(\alpha+i \beta, A)+R(-\alpha+i \beta, A)] .
\end{gathered}
$$

We have

$$
\begin{aligned}
D(\alpha+i \beta)- & D\left(\alpha+i \beta_{1}\right)= \\
= & (2 \alpha)^{-1}\left(\left[\alpha^{2}-\left(A-i \beta_{1}\right)^{2}\right]-\left[\alpha^{2}-(A-i \beta)^{2}\right]\right) D(\alpha+i \beta) D\left(\alpha+i \beta_{1}\right) \\
= & (2 \alpha)^{-1}\left[2 i\left(A-i \beta_{1}\right)\left(\beta_{1}-\beta\right)-\left(\beta_{1}-\beta\right)^{2}\right] D(\alpha+i \beta) D\left(\alpha+i \beta_{1}\right) \\
= & i\left(\beta_{1}-\beta\right)\left[R\left(\alpha+i \beta_{1}, A\right)+R\left(-\alpha+i \beta_{1}, A\right)\right] D(\alpha+i \beta) \\
& -\frac{\left(\beta_{1}-\beta\right)^{2}}{2 \alpha} D(\alpha+i \beta) D\left(\alpha+i \beta_{1}\right) .
\end{aligned}
$$

Since $\left(\beta_{1}-\beta\right)^{2} \alpha^{-1} \rightarrow 0$ and $\left|\beta_{1}-\beta\right| \cdot\left\|R\left(\alpha+i \beta_{1}, A\right)+R\left(-\alpha+i \beta_{1}, A\right)\right\|$ is bounded when $\alpha \rightarrow 0+, \alpha+i \beta_{1} \in \Omega^{h}(\beta)$, we conclude that

$$
\left\|D\left(\alpha+i \beta_{1}\right) x\right\| \rightarrow 0, \quad \alpha \rightarrow 0+, \alpha+i \beta_{1} \in \Omega^{h}(\beta) .
$$

(ii) Let $\lim _{\alpha \rightarrow 0+} D(\alpha+i \beta) x=y$. By (5.4),

$$
(A-i \beta) D(\alpha+i \beta) x=\alpha(R(\alpha+i \beta, A) x+R(-\alpha+i \beta, A) x) .
$$

Therefore, $D(\alpha+i \beta) x \in \operatorname{dom}(A-i \beta)^{2}$. Again by (5.4),

$$
(A-i \beta)^{2} D(\alpha+i \beta) x=-2 \alpha x+\alpha^{2} D(\alpha+i \beta) x \rightarrow 0, \quad \alpha \rightarrow 0+.
$$

Since $(A-i \beta)^{2}$ is closed, $y \in \operatorname{dom}(A-i \beta)^{2},(A-i \beta)^{2} y=0$ and then, by assumption, $y=0$.

(iii) Since $X$ is reflexive, the set $S:=\left\{D\left(\alpha_{n}+i \beta\right) x: n \in \mathbb{N}\right\}$ is weakly precompact for any sequence $\alpha_{n} \searrow 0, n \rightarrow \infty$. 
Let $y \in X$ be such that

$$
w-\lim _{n \rightarrow \infty} D\left(\alpha_{n_{k}}+i \beta\right) x=y
$$

for a subsequence $\alpha_{n_{k}} \searrow 0, k \rightarrow \infty$. As above,

$$
(A-i \beta)^{2} D\left(\alpha_{n_{k}}+i \beta\right) x=-2 \alpha_{n_{k}} x+\alpha_{n_{k}}^{2} D\left(\alpha_{n_{k}}+i \beta\right) x \rightarrow 0 .
$$

Since $(A-i \beta)^{2}$ is closed, it is also weakly closed, hence $(A-i \beta)^{2} y=0$, and then $y=0$. Thus the only weak (sequential) limit point of $S$ is 0 so that the weak limit of $D\left(\alpha_{n}+i \beta\right) x, n \rightarrow \infty$, is 0 . Since the choice of $\left\{\alpha_{n}\right\}$ was arbitrary, the assertion of the lemma follows.

Theorem 1.5 now follows from Theorem 4.2.

Proof of Theorem 1.5. We start with the proof of (1.4). Let

$$
X^{D}(F):=\left\{x \in X: \lim _{\alpha \rightarrow 0+} D(\alpha+i \beta) x=0, \beta \in \mathbb{R} \backslash F\right\} .
$$

Obviously, $x \in X(F)$ implies $x \in X^{D}(F)$ since the function $R(\lambda, A) x$ extends analytically through $i(\mathbb{R} \backslash F)$ by the definition of $\sigma(x)$. To prove the converse inclusion fix $x \in X^{D}(F)$ and note that

$$
\mathbb{R} \backslash F=\cup_{n \geq 1}\left(a_{n}, b_{n}\right), \quad-\infty \leq a_{n} \leq b_{n} \leq+\infty .
$$

Let further $x^{*} \in X^{*}$ and $n \in \mathbb{N}$ be fixed, and assume without loss of generality that $\left[i a_{n}, i b_{n}\right]$ is compact. The harmonic function $\left\langle D(\lambda) x, x^{*}\right\rangle$ defined on the rectangle $R_{n}=\left\{\lambda \in \mathbb{C}:-1 \leq \operatorname{Re} \lambda \leq 1, b_{n}<\operatorname{Im} \lambda<a_{n}\right\}$ satisfies the estimate (5.2). By Theorem 4.2 (b) (or rather its version for complex-valued harmonic functions) and Lemma $5.1(\mathrm{i}),\left\langle D(\lambda) x, x^{*}\right\rangle$ extends continuously to the interval $\left(i a_{n}, i b_{n}\right)$ and is zero there. Arguing as in the proof of Theorem 5.4 in [8] we obtain that $\left\langle R(\lambda, A) x, x^{*}\right\rangle$ extends analytically to $R_{n}$. Furthermore, by the uniform boundedness principle,

$$
\sup _{\lambda_{1}, \lambda_{2} \in R_{n} \backslash i \mathbb{R}, \lambda_{1} \neq \lambda_{2}}\left\|\frac{R\left(\lambda_{1}, A\right) x-R\left(\lambda_{2}, A\right) x}{\lambda_{1}-\lambda_{2}}\right\|<\infty,
$$

so that $R(\lambda, A) x$ is uniformly continuous on $R_{n} \backslash i \mathbb{R}$ and therefore extends continuously to $R_{n}$. The analyticity of $\left\langle R(\lambda, A) x, x^{*}\right\rangle$ in $R_{n}$ for every $x \in X^{*}$ implies that $R(\lambda, A) x$ is analytic in $R_{n} \backslash i \mathbb{R}$ so that $R(\lambda, A) x$ is analytic in $R_{n}$. Since $n$ was arbitrary, the proof is finished.

Let now $X$ be reflexive, $\sigma_{p}(A)=\emptyset$, and $a \in[0,2)$. Define

$$
X_{b}^{D}(F):=\left\{x \in X: \sup _{\alpha>0}\|D(\alpha+i \beta) x\|<\infty, \beta \in \mathbb{R} \backslash F\right\} .
$$

Then arguing as above gives $X(F) \subset X_{b}^{D}(F)$. Moreover, the argument used above shows that to verify the opposite inclusion it suffices to prove that for $x \in X_{b}^{D}$ 
and $x \in X^{*}$, the function $\left\langle D(\lambda) x, x^{*}\right\rangle$ satisfies the conditions of Theorem 4.2 for the approach function $h(t)=t^{a+1+\epsilon}, \epsilon \in(0,2-a)$. Since $\sup _{\alpha>0}\|D(\alpha+i \beta) x\|<\infty$, by Lemma 5.1 (iii) and the equality

$$
\begin{aligned}
& \left\langle\left(D(\alpha+i \beta)-D\left(\alpha+i \beta_{1}\right)\right) x, x^{*}\right\rangle= \\
& =i\left(\beta_{1}-\beta\right)\left\langle D(\alpha+i \beta) x,\left[R\left(\alpha+i \beta_{1}, A\right)+R\left(-\alpha+i \beta_{1}, A\right)\right]^{*} x^{*}\right\rangle \\
& \quad-\frac{\left(\beta_{1}-\beta\right)^{2}}{2 \alpha}\left\langle D(\alpha+i \beta) x, D\left(\alpha+i \beta_{1}\right)^{*} x^{*}\right\rangle
\end{aligned}
$$

obtained in the proof of Lemma 5.1 (i), it follows that $\left\langle D(\lambda) x, x^{*}\right\rangle$ satisfies (4.1). The verification of the condition (b) of Theorem 4.2 is straightforward, thus (1.5) is proved as well.

Remark 5.2. We do not know whether Theorem 1.5 is optimal with respect to the exponent $a$. While, by Proposition 1.4, we cannot have a counterpart of Theorem 4.2 in the situation where $a>2$, the corresponding examples of $C_{0}$-groups are still out of reach.

Proof of Theorem 1.6. First, we show that for every $n \in \mathbb{N}$,

$$
X(F) \subset \bigcap_{\beta \in \mathbb{R} \backslash F} \operatorname{ran}(i \beta-A)^{n} .
$$

If $x \in X(F)$, then the function $f(\lambda)=R(\lambda, A) x, \lambda \in \mathbb{C} \backslash i \mathbb{R}$, extends analytically to $\mathbb{C} \backslash i F$ as well as its derivatives, and we have

$$
f^{(k)}(\lambda)=(-1)^{k} k ! R^{k+1}(\lambda, A) x, \quad 1 \leq k \leq n .
$$

Since for any $\beta \in \mathbb{R} \backslash F$ and $\lambda \in \mathbb{C} \backslash i \mathbb{R}$,

$$
(i \beta-A)^{n} R^{n}(\lambda, A) x=x+\sum_{k=1}^{n} C_{n}^{k}(i \beta-\lambda)^{k} R(\lambda, A)^{k} x,
$$

by the closedness of $(i \beta-A)^{n}$ we obtain

$$
(i \beta-A)^{n} f(i \beta)=x, \quad \beta \in \mathbb{C} \backslash F,
$$

so that

$$
x \in \bigcap_{\beta \in \mathbb{R} \backslash F} \operatorname{ran}(i \beta-A)^{n} .
$$

To prove the equality in (1.7), we note that $x \in \operatorname{ran}(i \beta-A)^{3}, \beta \in \mathbb{R} \backslash F$, imply for $a \in[1,2)$ that $\lim _{\alpha \rightarrow 0+} D(\alpha+i \beta) x=0$. Indeed, if $x=(i \beta-A)^{3} y$, then, by (5.3) and (5.4),

$$
\begin{aligned}
& D(\alpha+i \beta)(A-i \beta)^{3} y=\alpha^{2} D(\alpha+i \beta)(A-i \beta) y-2 \alpha(A-i \beta) y \\
& \quad=\alpha^{3}[R(\alpha+i \beta, A)+R(-\alpha+i \beta, A)] y-2 \alpha(A-i \beta) y \rightarrow 0+, \quad \alpha \rightarrow 0+.
\end{aligned}
$$

Then, by Theorem $1.5, R(\lambda, A) x$ extends analytically to $\mathbb{C} \backslash F$, and the statement follows. The proof of (1.6) is analogous. 
Remark 5.3. In fact, to prove (1.6) we could just use Theorem 1.3 (a), and deal only with the radial behavior of the resolvents.

Remark 5.4. Observe that in (1.6), (1.7) one cannot replace $\operatorname{ran}(i \beta-A)^{n}$, $n=2,3$, by $\operatorname{ran}(i \beta-A)$, see page 136 of [4]. We do not know, however, whether one can replace the exponent 2 in (1.6) by a real number smaller than 2 .

Theorems 1.5 and 1.6 apply in particular to $C_{0}$-groups with (at most) linear growth. As a natural example of such groups we mention $C_{0}$-groups $(\mathcal{T}(t))_{t \in \mathbb{R}}$ on a Banach space $\mathcal{X}=X \oplus X$ generated by the triangular operator matrices of the form

$$
\mathcal{A}=\left(\begin{array}{cc}
A_{1} & B \\
O & A_{2}
\end{array}\right)
$$

where $A_{1}$ and $A_{2}$ are the generators of bounded $C_{0}$-groups $\left(T_{1}(t)\right)_{t \in \mathbb{R}}$ and $\left(T_{2}(t)\right)_{t \in \mathbb{R}}$ on $X$, and $B$ is bounded from $\operatorname{dom}\left(A_{2}\right)$ to $\operatorname{dom}\left(A_{1}\right)$. The operator $\mathcal{A}$ can be treated as an off-diagonal perturbation of the generator of a bounded $C_{0}$-group defined by the matrix with diagonal entries $A_{1}$ and $A_{2}$. The corresponding semigroup $(\mathcal{T}(t))_{t \geq 0}$ is given by

$$
\mathcal{T}(t)=\left(\begin{array}{cc}
T_{1}(t) & \int_{0}^{t} T(s) B T(t-s) d s \\
O & T_{2}(t)
\end{array}\right), \quad t \geq 0 .
$$

Such matrices appear frequently in applications, e.g., in the study of second order abstract and concrete Cauchy problems. For more details on this subject, see e.g. $[32]$.

Note that results similar to Theorems 1.5 and 1.6 hold also for discrete groups $\left(T^{n}\right)_{n \in \mathbb{Z}} \subset \mathcal{L}(X)$ such that $\left\|T^{n}\right\| \leq M(|n|+1)^{a}, a \in[0,2), n \in \mathbb{Z}$. Their proofs are straightforward modifications of the proofs of Theorems 1.5 and 1.6 using the same boundary uniqueness statements of Theorem 4.2. We leave formulation of these results to the interested reader.

Acknowledgment. The authors are grateful to the referee for useful comments and suggestions which led to an improvement of the paper.

\section{References}

[1] Alvarez, J., Guzmán-Partida, M. and Pérez-Esteva, S.: Harmonic extensions of distributions. Math. Nachr. 280 (2007), 1443-1466.

[2] Amrein, W. O., Boutet de Monvel, A. And Georgescu, V.: C $C_{0}$-groups, commutator methods and spectral theory of $N$-body Hamiltonians. Progress in Mathematics 135, Birkhäuser Verlag, Basel, 1996.

[3] Baillet, M.: Analyse spectrale des opérateurs hermitiens d'une espace de Banach. J. London Math. Soc. (2) 19 (1979), 497-508.

[4] Batty, C. J. K., Chill, R. And Tomilov, Yu.: Strong stability of bounded evolution families and semigroups. J. Funct. Anal. 193 (2002), 116-139.

[5] Baumgärtel, H. And Wollenberg, M.: Mathematical scattering theory. Operator Theory: Advances and Applications 9, Birkhäuser Verlag, Basel, 1983. 
[6] Borichev, A.: Beurling algebras and the generalized Fourier transform. Proc. London Math. Soc. (3) 73 (1996), 431-480.

[7] Borichev, A.: On the minimum of harmonic functions. J. Anal. Math. 89 (2003), 199-212.

[8] Borichev, A., Chill, R. And Tomilov, Yu.: Uniqueness theorems for (sub-)harmonic functions with applications to operator theory. Proc. London Math. Soc. (3) 95 (2007), 687-708.

[9] Brennan, J. E. And Volberg, A. L.: Asymptotic values and the growth of analytic functions in spiral domains. Publ. Mat. 37 (1993), 465-477.

[10] Brossard, J. and Chevalier, L.: Problème de Fatou ponctuel et dérivabilité des mesures. Acta Math. 164 (1990), 237-263.

[11] Domar, Y.: On the existence of a largest subharmonic minorant of a given function. Ark. Mat. 3 (1958), 429-440.

[12] Donoghue, W. F, Jr.: A theorem of the Fatou type. Monatsh. Math. 67 (1963), 225-228.

[13] Curtis, Ph. C., JR. And Neumann, M.: Nonanalytic functional calculi and spectral maximal spaces. Pacific J. Math. 137 (1989), 65-85.

[14] Foiaş, C. And Vasilescu, F.-H.: Non-analytic local functional calculus. Czechoslovak Math. J. 24 (1974), 270-283.

[15] Garnett, J. B. And Marshall, D. E.: Harmonic measure. New Mathematical Monographs 2, Cambridge University Press, Cambridge, 2005.

[16] Gehring, F. W.: The Fatou theorem and its converse. Trans. Amer. Math. Soc. 85 (1957), 106-121.

[17] JøRgensen, P. E. T.: Spectral theory for infinitesimal generators of one-parameter groups of isometries: the min-max principle and compact perturbations. J. Math. Anal. Appl. 90 (1982), 343-370.

[18] JøRgensen, P.E.T.: Spectral theory for one-parameter groups of isometries. J. Math. Anal. Appl. 168 (1992), 131-146.

[19] Kantorovitz, S.: Spectral theory of Banach space operators. $C^{k}$-classification, abstract Volterra operators, similarity, spectrality, local spectral analysis. Lecture Notes in Mathematics 1012, Springer-Verlag, Berlin, 1983.

[20] KocAn, D.: Spectral manifolds for a class of operators. Illinois J. Math. 10 (1966), 605-622.

[21] Kocan, D.: A characterization of some spectral manifolds for a class of operators. Illinois J. Math. 16 (1972), 359-369.

[22] Koosis, P.: The logarithmic integral. I. Cambridge Studies in Advanced Mathematics 12, Cambridge University Press, Cambridge, 1988.

[23] Kritt, B.: A theory of unbounded generalized scalar operators. Proc. Amer. Math. Soc. 32 (1972), 484-490.

[24] KRITT, B.: The Fourier transform of an unbounded spectral distribution. Proc. Amer. Math. Soc. 35 (1972), 74-80.

[25] Laursen, K. B. And Neumann, M.: An introduction to local spectral theory. London Mathematical Society Monographs, New Series, 20, The Clarendon Press, Oxford University Press, New York, 2000.

[26] Loomis, L. H.: The converse of the Fatou theorem for positive harmonic functions. Trans. Amer. Math. Soc. 53 (1943), 239-250. 
[27] Marschall, E.: Funktionalkalküle für abgeschlossene lineare Operatoren in Banachräumen. Manuscripta Math. 35 (1981), 277-310.

[28] Marschall, E.: On the functional-calculus of nonquasianalytic groups of operators and cosine functions. Rend. Circ. Mat. Palermo (2) 35 (1986), 58-81.

[29] Miller, T. L., Miller, V. G. and Neumann, M.: Spectral subspaces of subscalar and related operators. Proc. Amer. Math. Soc. 132 (2004), 1483-1493.

[30] Müller, V.: Spectral theory of linear operators and spectral systems in Banach algebras., second edition. Operator Theory: Advances and Applications 139, Birkhäuser Verlag, Basel, 2007.

[31] Nagel, A., Rudin, W. And Shapiro, J. H.: Tangential boundary behavior of functions in Dirichlet-type spaces. Ann. of Math. (2) 116 (1982), 331-360.

[32] Nagel, R.: Towards a "matrix theory" for unbounded operator matrices. Math. Z. 201 (1989), 57-68.

[33] Nikol'skil, N. K.: Selected problems of weighted approximation and spectral analysis. Trudy Mat. Inst. Steklov. 120, Izdat. "Nauka" Leningrad. Otdel., Leningrad, 1974. English translation in Procedings of the Steklov Institute of Mathematics, 120, 1974. American Mathematical Society, Providence, RI, 1976.

[34] Rudin, W.: Tauberian theorems for positive harmonic functions. Nederl. Akad. Wetensch. Indag. Math. 40 (1978), 376-384.

[35] Shapiro, V.L.: The uniqueness of functions harmonic in the interior of the unit disk. Proc. London Math. Soc. (3) 13 (1963), 639-652.

[36] Vagharshakyan, A.: On the maximum principle for harmonic functions. Algebra $i$ Analiz 20 (2008), no. 3, 1-17. Translation in St. Petersburg Math. J. 20 (2009), 325-337.

[37] VRbová, P: Structure of maximal spectral spaces of generalized scalar operators. Czechoslovak Math. J. 23 (1973), 493-496.

[38] Warschawski, S. E.: On conformal mapping of infinite strips. Trans. Amer. Math. Soc. 51 (1942), 280-335.

[39] Wolf, F.: The Poisson integral. A study in the uniqueness of harmonic functions. Acta. Math. 74 (1941), 65-100.

Received January 29, 2010; revised June 16, 2010.

Alexander Borichev: Centre de Mathématiques et Informatique, Université d'AixMarseille I, 39 rue Frédéric Joliot-Curie, 13453 Marseille, France.

E-mail: borichev@cmi.univ-mrs.fr

Yuri Tomilov: Department of Mathematics and Computer Science, Nicolas Copernicus University, ul. Chopina 12/18, 87-100 Torun, Poland and Institute of Mathematics, Polish Academy of Sciences, Śniadeckich str. 8, 00-956 Warsaw, Poland.

E-mail: tomilov@mat.uni.torun.pl

This work was completed with the support of the Marie Curie Transfer of Knowledge program, project "TODEQ". The first author was also partially supported the ANR projects DYNOP and FRAB. The second author was partially supported the MNiSzW grant Nr. N201384834. 